\title{
Prácticas de inclusión escolar en el departamento de Sonsonate Estudio piloto: Centro Escolar República de Haití
}

\section{School inclusion practices in the department of Sonsonate: A Pilot study at Centro Escolar República de Haití}

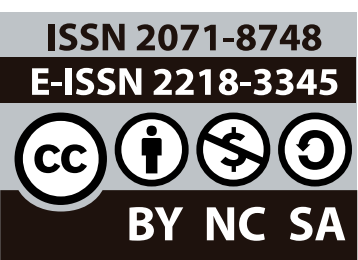

DOI: https://doi.org/10.5377/entorno.v0i69.9558 URI: http://hdl.handle.net/11298/1152

\author{
Saúl Campos-Morán \\ Investigador Utec \\ saul.campos@utec.edu.sv \\ ORCID 0000-0002-8884-5547 \\ Paola María Navarrete \\ Investigadora Utec \\ Ana María Zelidón \\ Investigadora USO \\ Maynor Guillermo Reynado \\ Investigador UTLA \\ Recibido: 17 de febrero 2020 \\ Aceptado: 27 de junio de 2020
}

\section{Resumen}

Elobjetivo de la investigaciónfue identificar el conocimiento actual, las estrategias implementadas, los problemas de actitud y capacitación encontrados en el Centro Escolar República de Haití sobre problemas de aprendizaje en el aula de clase para fomentar la inclusión social y cognitiva de los niños con problemas de aprendizaje. Para ello se aplicó un diseño transversal del tipo descriptivo. El estudio estuvo compuesto de dos etapas. La primera, a la que se le denominó Fase Cuantitativa, la cual se realizó a través de la observación descriptiva mediante criterio arbitrario de observación; y la segunda, Fase Cualitativa, en la que se realizaron entrevistas semiestructuradas.

Se recopiló información de estudiantes con indicios de dificultades del aprendizaje a través de los docentes de la institución en estudio. Los docentes con los que se

\section{Abstract}

The objective of this research was to identify the present knowledge, the implemented strategies, the attitude problems and the training found at the Centro Escolar República de Haití in relation to the learning problems inside the classroom in order to promote the social and cognitive inclusion of children with learning problems/ disabilities. With this in mind, a transversal descriptive design was applied. The study was conducted in two stages. The first one, called Quantitative Phase, was conducted via descriptive observation through an arbitrary observation criteria; the second one, the Qualitative Phase, used semistructured interviews.

The faculty from the primary school facilitated data about those students with learning difficulties. 
trabajó fueron del nivel básico, tanto de primero como del segundo ciclo.

Entre los resultados principales que se obtuvieron, estuvo el hecho de que existe una opinión negativa de algunos docentes para que niños con problemas y/o trastornos de aprendizaje asistan a la escuela regular; y esto debido a que no se les puede atender adecuadamente, aspecto que guarda relación con la necesidad de capacitación en gestión y manejo efectivo de niños con trastornos de aprendizaje en el aula, así como la apatía de algunos de ellos para hacerlo. En relación con la caracterización de los niños, se puede apreciar que existen problemas de aprendizaje en las áreas de comunicación oral y escrita, lectura, matemática, aspectos cognitivos, presentación y orden, así como habilidades psicomotrices.

\section{Palabras clave}

Educación Inclusiva - Sonsonate, El Salvador; Psicopedagogía - Sonsonate, El Salvador; Personas con problemas de aprendizaje - Sonsonate, El Salvador; Psicología del aprendizaje; Trastornos del aprendizaje. Neuropsicología del aprendizaje.

El Salvador es un país cuya inclusión escolar es incipiente, lo cual contrasta con la demanda mundial (Muñoz, 2019). La Política de Educación Inclusiva en El Salvador fue creada en respuesta a necesidades de quienes no acceden a la escuela, los que no avanzan como se espera, los repitentes, estudiantes con sobreedad u otras condiciones, y para aquellos que no pueden continuar con sus estudios en el grado inmediato superior o el siguiente nivel (Ministerio de Educación, 2010) Sin embargo, no existe hasta la fecha un diagnóstico de su correcta implementación en alguna de las dependencias destinadas a darle cumplimiento.

El proceso de investigación se realizó en el Centro Escolar República de Haití del municipio de Sonsonate, departamento de Sonsonate. La institución seleccionada es estratégica para la investigación por la inmersión que tienen los docentes de la institución educativa en el trabajo con niños, niñas y adolescentes con necesidades educativas especiales.
Among the main results, there can be mentioned the fact that some faculty members had a negative opinion about students with learning difficulties and/or disabilities attending the regular school since they cannot be adequately assisted, an aspect which relates to the need in training on how to effectively manage children with learning disabilities inside the classroom, in addition to the apathy that they have to do it. In relation to the characterization of children, it can be appreciated that there are learning problems in the areas of verbal and written communication, reading, mathematics, cognitive aspects, personal appearance and care, and organization as well as psychomotor skills.

\section{Keywords}

Scientific dissemination-San Luis Potosí, Mexico. Science and technology - San Luis Potosí, Mexico. Scientific research - San Luis Potosí, Mexico. Social research - San Luis Potosí, Mexico.

\section{Figura 1: Ubicación geográfica COED. República de Haití}

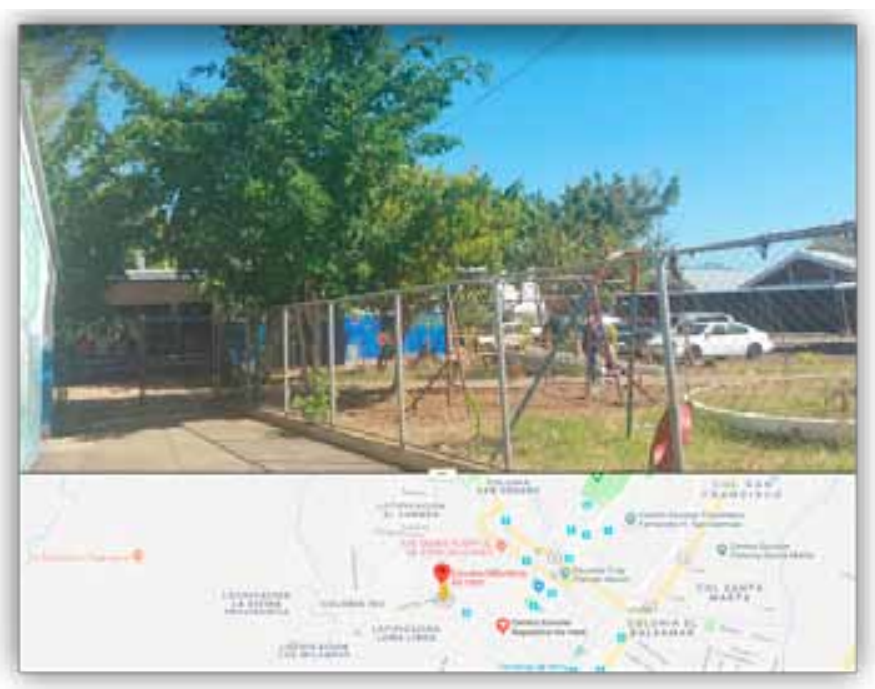

Fuente: Google Maps. 


\section{Inclusión escolar}

La Organización de las Naciones Unidas para la Educación, la Ciencia y la Cultura define la inclusividad como "el proceso de identificar y responder a la diversidad de las necesidades de todos los estudiantes a través de la mayor participación en el aprendizaje, las culturas y las comunidades, y reduciendo la exclusión en la educación. Involucra cambios y modificaciones en contenidos, aproximaciones, estructuras y estrategias, con una visión común que incluye a todos los niño/as del rango de edad apropiado y la convicción de que es la responsabilidad del sistema regular, educar a todos los niño/as" (Inclusión International, s.f.)

Desde la Declaración Universal de los Derechos Humanos, en 1948, se afirmó el derecho universal a una educación gratuita y obligatoria, al menos en los niveles primarios, para brindar a toda la población acceso a la educación (Crosso, 2014). Si bien la educación es un derecho fundamental para cada uno de los salvadoreños, en América Latina el acceso que tiene esta población con discapacidad a este derecho es limitado (Muñoz, 2011).

La inclusión educativa no es fácil de implementar, exige una gran cantidad de cambios dentro del sistema educativo y en la sociedad misma, requiriendo una comprensión global desde un modelo biopsicosocial (Organización de las Naciones Unidas [ONU], 2007).

Según cifras del Banco Mundial, en América Latina y el Caribe solo del 20 al $30 \%$ de niños con discapacidad asisten a la escuela, ya que suelen ser excluidos de los sistemas educativos, ya sea por accesibilidad o formas de enseñanza. A pesar de esta evidente necesidad que tiene la población con discapacidad al ingresar a los sistemas educativos, es escasa la información estadística disponible sobre índices de abandono o deserción escolar de niños, niñas y adolescentes con discapacidad, y casi inexistente la comparación estadística de estos índices con alumnos sin discapacidad, o incluso del éxito o fracaso escolar de dicha población (Crosso, (2014). Las repercusiones de esta falta de inclusión son, para Muñoz (2011), fáciles de prever, ya que la educación es la puerta de entrada para lograr la inclusión en otras áreas, tales como la social y la laboral.

\section{¿Neuropsicología del aprendizaje?}

Hablar de Neuropsicología del aprendizaje implica integrar tres componentes: neurociencias, psicología y aprendizaje (Ecklund-Johnson, 2019).
Las neurociencias aportan el componente biológico, de ciencias naturales, necesario para comprender los sustratos neuronales involucrados en el proceso de aprendizaje (Muñoz, 2011). La Psicología estudia el comportamiento, y en este caso aporta el conocimiento de la conducta de quien está en un proceso formativo o de quien aprende. Ahora, la Neuropsicología en sí misma es una disciplina que hace parte de las neurociencias y en la cual se estudia la relación de los problemas neurológicos (traumas, accidentes cerebrovasculares, tumores, enfermedades neurodegenerativas y del neurodesarrollo, epilepsia, entre otros) con el efecto que puede tener en el comportamiento individual y en los procesos cognitivos y emocionales; en otras palabras, y como su nombre lo indica, es la unión entre las neurociencias y la psicología, y, por tanto, estudia la relación entre el cerebro y la conducta (Arismendi, Primero y Tabullo, 2005).

La Asociación Americana de Psicología (APA, siglas del inglés) define la Neuropsicología como "la especialidad en psicología que aplica los principios de evaluación e intervención basados en los estudios científicos del comportamiento humano y que se relacionan con la normalidad o anormalidad del funcionamiento del sistema nervioso central; la neuropsicología se dedica a mejorar la comprensión de la relación entre el cerebro y el comportamiento y la aplicación de ese conocimiento al ser humano" (APA, 2010).

Estas tres áreas del conocimiento son fundamentales para la atención integral de niños y jóvenes con dificultades en el aprendizaje, pues desde el componente neurocien ${ }^{+i f i}{ }^{\prime}$ o se aborda la forma como aprende el cerebro desde na perspectiva o enfoque local-conexionista (Capilla, 2011). Desde el componente psicológico, se analiza la forma como impactan a nivel socioemocional y comportamental los diferentes trastornos del aprendizaje o trastornos que tienen incidencia en el rendimiento académico; y desde el componente educativo se analiza la forma como se pueden implementar estrategias educativas, a fin de que el niño o adolescente mejore su capacidad de aprender [percepción, atención, memoria, concienciación] (Ecklund-Johnson, 2019).

En el caso de El Salvador, se cuenta con un programa de educación inclusiva, el cual tiene como escuela insignia el Centro Escolar República de Haití, donde se llevará a cabo la investigación. El objetivo de este programa es brindar oportunidades de acceso, permanencia y de aprendizaje 
efectivo, en todos los niveles educativos, a estudiantes con necesidades individuales especiales o en situación de riesgo social, y al 2019 tiene como principal logro la incorporación de 2.004 niños y niñas con sobreedad, en riesgo social y/o fuera del sistema educativo a través de la modalidad de educación acelerada de segundo a sexto grado en 2018, y 1.627 niñas y niños (Mined, 2019).

De tal manera, el objetivo general de la investigación fue diagnosticar, por medio de la identificación del conocimiento actual, las estrategias implementadas y los problemas encontrados en el Centro Escolar República de Haití sobre problemas de aprendizaje en el aula de clase para fomentar la inclusión social y cognitiva de los niños, niñas y adolescentes con problemas de aprendizaje. Para tal efecto, se buscó cumplir los siguientes objetivos específicos:

1. Analizar el grado de conocimiento que existe sobre la inclusión escolar y los traumas y trastornos del aprendizaje de los docentes que laboran en el Centro Escolar República de Haití.

2. Contrastar las estrategias pedagógicas que han sido implementadas en la institución educativa para atender a los niños, niñas y adolescentes con discapacidad cognitiva.

3. Establecer una caracterización de los niños, niñas y adolescentes que presentan características propias de los trastornos específicos de aprendizaje.

El estudio estuvo orientado a determinar la forma como se debe reorientar la educación, para darle cabida a la inclusión educativa a través de un cambio de paradigma en la educación. De tal manera, nos orientamos a la realización de un diagnóstico sobre el impacto que pueden tener los trastornos del aprendizaje en el aula de clase, abordando la problemática desde un enfoque de inclusión escolar en El Salvador e identificando los efectos que puede tener en el desarrollo de los estudiantes dentro de las aulas.
En la investigación, nos centramos en la identificación de factores que pueden ser determinantes para hacer que una institución educativa sea inclusiva. Así, se analizaron aspectos como la importancia percibida de los docentes en la inclusión educativa, las prácticas que se desarrollan en el aula de clase cuando se ha identificado que un niño, niña 0 adolescente requiere de planes educativos especiales; $y$ las políticas institucionales que se han desarrollado para que el ambiente educativo sea favorable para superar las dificultades cognitivas.

\section{Tipo de estudio}

Se procedió según el modelo de Montero, \& León (2007) de estudios de tipo descriptivo con diseño transversal. El estudio estuvo compuesto de las siguientes dos etapas:

Fase cuantitativa: Observación descriptiva mediante criterio arbitrario de observación

Fase cualitativa: Entrevista semiestructurada

Durante la primera fase (cuantitativa) se aplicaron instrumentos validados a la realidad salvadoreña por un equipo local de psicólogos que permitió garantizar, por medio de procedimientos de validez de contenido y constructo, y de confiabilidad, aplicar un instrumento descriptivo que tuvo como propósito identificar indicadores de presencia de alguno de los trastornos que se persiguió identificar por medio de la investigación. El resultado de esta parte nos dio datos descriptivos que pueden generalizarse a la institución educativa. Durante la segunda fase (cualitativa) se aplicaron los instrumentos de entrevista semiestructurada.

\section{Variables con su definición conceptual}

En la siguiente tabla se detalla la conceptualización de las variables contempladas para este estudio.

\section{Tabla 1. Conceptualización de variables}

\begin{tabular}{ll}
\hline Inclusión escolar/Educativa & Es el proceso de identificar y responder a la diversidad de las necesidades de todos los \\
& estudiantes con el fin de facilitar su aprendizaje exitoso. \\
\hline Neuropsicología del aprendizaje & Área de las neurociencias que se encarga de estudiar los problemas que interfieren con \\
& el proceso de aprendizaje desde una perspectiva neurocientífica (Centro del Pensamiento \\
& en Redes Neuroeducatrónicas). \\
\hline
\end{tabular}

Fuente: Elaboración propia. 


\section{Población y muestra}

La naturaleza del estudio implica un abordaje cuanticualitativo significando que no se trabajó con un universo calculado, sino que se abordó el centro escolar como piloto donde se aplicaron pruebas de diagnóstico y se hizo un análisis completo de la forma como en la institución educativa se trabaja con los estudiantes a quienes se han identificado con dificultades del aprendizaje en el Centro de Recursos para la Inclusión (CRI). En la tabla 2 se enuncian las características de los participantes en el estudio.

La población participante fue de 10 docentes. De estos, se observa que $70 \%$ fue del género masculino, mientras que $30 \%$ del masculino. Entre las carreras de los participantes, se aprecia que los docentes que atienden a los niños están graduados de licenciaturas en Lenguaje y Literatura,

Tabla 2. Caracterización de docentes participantes

\begin{tabular}{llcc}
\hline & & Frecuencia & \% del N de columna \\
\hline \multirow{2}{*}{ Género } & Femenino & 7 & 30,0 \\
& Masculino & 30,0 & 1 \\
& Licenciatura en Lenguaje y Literatura & 1 & 1 \\
& Licenciatura en Educación Parvularia & 4 & 1 \\
Profesión & Licenciatura en Matemática & 1 & 1 \\
& Profesorado General & 1 & 14,3 \\
& Profesorado en Matemáticas & 1 & 28,6 \\
& Profesorado en Educación Física & 2 & 14,3 \\
Año de graduación & Licenciatura en Educación Básica & 1 & 42,9 \\
\hline
\end{tabular}

Fuente: Elaboración propia.

Tabla 3. Características de los sujetos de educación básica

Características de inclusión Características de exclusión

Entre 8 y 12 años de edad (etapa de desarrollo de niñez/ Niños menores de 7 años de edad antes de la pubertad)

Tanto del sexo masculino como femenino Estudiantes de escuelas privadas

Matriculados y activos en cualquiera de los grados correspondientes a su edad en el sector público salvadoreño

Que cuenten con autorización de sus padres para la participación en el estudio

Fuente: Elaboración propia.
Educación Parvularia, Matemática, Profesorado, y Licenciatura en Educación Básica. Respecto al año de su graduación, el valor más reciente que se aprecia es del 2000 , lo cual implica por lo menos 19 años de servicio en educación, asumiendo que existió trabajo activo desde el momento de su egreso de la universidad. El valor más antiguo es de 1981, mostrado por una persona, lo cual indica 38 años de servicio como docente.

\section{Sujetos}

La recopilación de información de los estudiantes con indicios de dificultades del aprendizaje se realizó a través de los docentes del centro Escolar República de Haití, el cual fue la sede en la que el trabajo de campo se llevó a cabo. Los docentes con los que se trabajó fueron del nivel básico, tanto de primero como de segundo ciclo. Los criterios para seleccionarlos se detallan en la tabla 3. 
Así, se garantizó la participación de los estudiantes de conformidad con lo mandado por la Ley Ley de Protección Integral de la Niñez y Adolescencia en sus artículos correspondientes.

\section{Caracterización de los niños participantes}

Esta sección obedece al segundo cuestionario, el cual estuvo dirigido a caracterizar los trastornos de los niños según la percepción de los adultos encargados de su proceso de enseñanza.

\section{Procedencia y diagnóstico previo}

El $45 \%$ de los participantes no pudo ser identificado acerca de su municipio de residencia, pero del resto, el 36,4\% proviene de Sonsonate, $9.1 \%$ de Nahuizalco y $9,1 \%$ de San Antonio.

En cuanto a diagnósticos previos, el $80 \%$ de los niños, respecto a los que se llenó el instrumento, ya contaban con un diagnóstico, mientras que un $20 \%$ no contaba con dicha certificación, por lo que su condición de trastorno es

Tabla 4. Características generales de los estudiantes participantes

\begin{tabular}{lccc}
\hline & & Recuento & \% del N de columna \\
\hline \multirow{4}{*}{ Edad } & 9 & 2 & 20 \\
& 10 & 2 & 20 \\
& 12 & 2 & 20 \\
\hline \multirow{3}{*}{ Género } & 14 & 2 & 20 \\
& 15 & 1 & 10 \\
\hline \multirow{3}{*}{ Grado que cursa } & 17 & 1 & 10 \\
& Masculino & 5 & 50,0 \\
& Femenino & 5 & 50,0 \\
& 1 & 1 & 9,1 \\
\hline \multirow{3}{*}{ Grados reprobados } & 2 & 2 & 18,2 \\
& 3 & 3 & 27,3 \\
& 6 & 3 & 27,3 \\
& 7 & 1 & 9,1 \\
& 0 & 1 & 16,7 \\
\hline
\end{tabular}

Debido a la naturaleza del centro escolar, no es inusual encontrar estudiantes que tengan una edad superior a la media normal para los grados de educación básica. Así, podemos observar edades que rondan entre los 9 y los 17 años para los participantes del estudio. De estos, fueron $50 \%$ niñas y $50 \%$ niños, los cuales cursan entre 1.0 y 8.0 grado. Un dato importante es que todos los participantes reprobaron por lo menos un grado durante su estancia formativa en el centro escolar. un diagnóstico empírico de los docentes que trabajan con el estudiante. Entre los que sí cuentan con ese diagnóstico, se identificaron enfermedades tales como hiperactividad, retraso mental y síndrome de Down.

\section{Procedimiento y métodos de recolección de datos}

Primero se validaron los instrumentos para la fase 1 con un equipo de psicólogos. A partir de ello, se contactó con la dirección del centro educativo que se intervendría, 
Tabla 5. Municipio de procedencia y diagnóstico previo de trastornos

\begin{tabular}{|c|c|c|c|}
\hline & & Recuento & $\%$ del $\mathrm{N}$ de columna \\
\hline \multirow{4}{*}{ Municipio de residencia } & & 4 & 45,5 \\
\hline & Nahuizalco & 1 & 9,1 \\
\hline & San Antonio & 1 & 9,1 \\
\hline & Sonsonate & 4 & 36,4 \\
\hline \multirow{2}{*}{ ¿Ha sido diagnosticado? } & Sí & 8 & 80,0 \\
\hline & No & 2 & 20,0 \\
\hline \multirow{5}{*}{$\begin{array}{l}\text { Si aplica, ¿cuál es el } \\
\text { diagnóstico? }\end{array}$} & & 4 & 45,5 \\
\hline & Hiperactivo & 3 & 27,3 \\
\hline & Retardo & 1 & 9,1 \\
\hline & Síndrome de Down & 1 & 9,1 \\
\hline & Sordo Total & 1 & 9,1 \\
\hline
\end{tabular}

lo cual se realizó a partir de la de gestión directa que las universidades hicieron con dichos centros. A partir de ello, se estableció una reunión con la Dirección, con el fin de presentar el estudio y gestionar un grupo focal con el equipo de trabajo para presentar el instrumento por aplicar y el procedimiento que se seguiría para la recolección de información. Luego, una vez aprobado el procedimiento por parte de las autoridades correspondientes, el equipo investigador, de forma directa, aplicó el instrumento en la institución educativa seleccionada. Se contó con un equipo de trabajo de apoyo que permitió controlar la aplicación de la prueba en relación con el número de docentes presentes en el centro escolar.

\section{Procedimiento y análisis de datos}

Luego de aplicar los instrumentos, se procedió a su respectiva tabulación por medio de los programas computacionales: SPSS versión 21, y R, el cual cuenta con un módulo cualitativo para preguntas abiertas. La tabulación estuvo a cargo de personal asistente (estudiantes ayudantes de investigación), luego el equipo investigador generó los resultados descriptivos para cada caso y de forma global. Se aplicó un barrido en los datos que permitió la caracterización de casos en los cuales se tenía indicios de encontrarse una dificultad en el aprendizaje. Una vez identificados los casos, se llevó a cabo una reunión de presentación con la Dirección del centro escolar.

\section{Análisis y discusión de resultados}

Percepción de la pertinencia de la asistencia de niñez con capacidades especiales a la escuela

En este apartado se sondeó la percepcióngeneral del impacto que la atención a niños con trastornos de aprendizaje tiene en el proceso de enseñanza de los docentes. Los resultados de la sección sugieren una opinión negativa por parte de los docentes en relación con la asistencia a la escuela de niños con problemas de aprendizaje. Para la cuestión de si estos deberían asistir a la escuela, la opinión positiva es solo de $61,5 \%$. A esto se le suma la cuestión de que si la inclusión de dichos niños tiene consecuencias negativas para el proceso de enseñanza-aprendizaje de los demás niños, en lo cual un $38,5 \%$ está de acuerdo. Asimismo, y en una opinión más dividida, se aprecia que existe una percepción de que estar con niños que no tienen trastornos puede generar impactos negativos en los niños que sí los tienen, con una relación de $50 \%$.

Finalmente, existe una marcada preocupación por los impactos que el bullying pueda tener en los niños con trastornos de aprendizaje por parte de los niños que no los tienen, con un $61,5 \%$ de docentes opinando que este puede ser un motivo de peso para evitar que los niños vayan a la escuela, en primer lugar. 


\section{Percepción de necesidades capacitación docente}

El sondeo de necesidades de capacitación está vinculado con las falencias percibidas a nivel individual y colectivo con relación a las competencias necesarias para la gestión y el manejo efectivo de niños con trastornos de aprendizaje en el aula, así como la gestión de la relación de estos con sus pares que carecen de dichos trastornos. De tal manera, no se midió o preguntó sobre competencias docentes genéricas, puesto que se asume que los docentes participantes cuentan con un mínimo de dichas habilidades. Aquí se aprecia que la mayoría de docentes que trabajan con niños con trastornos de aprendizaje opinan que un profesor no debería atender en el aula a un estudiante en dicha situación, con un $61,5 \%$ compartiendo dicha opinión. En contraste, se aprecia que un $53,8 \%$ piensa que son los profesores los que deben asumir la responsabilidad de enseñar a dichos niños, lo cual sugiere que la percepción de la pregunta inicial no se refiere a la no voluntad, sino, más bien, a una falta de capacitación, lo cual se comprueba con los ítems siguientes.

Para la pregunta de si se cree que existen niños con discapacidades cognitivas que aún no han sido identificados, un $76,9 \%$ opina que podría ser el caso, y un $100 \%$ apoya la noción de que es necesario capacitarse para poder identificar de forma temprana cualquier manifestación de dificultades del aprendizaje en los estudiantes.

\section{Capacitaciones}

Del total de docentes, la mayoría, un 61,5\%, se ha capacitado en cómo identificar trastornos de aprendizaje. Eso deja un $38,5 \%$ de profesores entrevistados que no cuentan con dicho entrenamiento, y por lo tanto es posible asumir que no cuentan con las competencias necesarias para hacer dicha identificación. En la misma línea, en cuanto a la atención, solamente el 53,8 \% cuenta con formación especializada para atender a estudiantes con trastornos especiales, lo cual sugiere que el resto de participantes solamente cuenta con formación de carácter empírico. Asimismo, se aprecia que sí se ha recibido formación en cuanto a inclusión educativa, pero no se hace la asociación entre ambos temas, siendo un $76,9 \%$ los que manifiestan haber recibido formación en dicho tema. Paralelamente, las capacitaciones que dicen sí haber recibido, incluyen los temas de Pedagogía y Didáctica, tecnologías de la información y comunicación, tecnología educativa y áreas básicas. Se aprecia que nadie mencionó capacitación en las áreas de Ética, Educatrónica, Neuropsicología del aprendizaje o Psicología educativa.

Al contrastar esta información y preguntar directamente si existiría disposición de capacitarse en el área de dificultades de aprendizaje y trastornos psicológicos, solamente el 69,2 \% manifestó estar interesado, valor que se repite al preguntar si habría disposición de capacitarse en inclusión educativa. Este mismo grupo opinó que deber ser prioridad para los docentes capacitarse en estas áreas.

Recurrencia de casos de problemas académicos en estudiantes

Al indagar si ha existido algún caso de bajo rendimiento académico asociado con factores como alimentación, falta a clases, desescolarización o enfermedades largas, se encontró que un $61,5 \%$ de los participantes tiene o ha tenido un caso con dichas características.

Entre los casos identificados, se encuentran los siguientes:

- El estudiante no logra resolver las tareas aun cuando estuvo en clase.

- Atraso en clases debido a falta por enfermedad.

- Un niño con problemas de habla.

- Caso de niño con síndrome de Down.

- Alumnos con problemas neurológicos de nacimiento.

- Hiperactividad.

- Retraso leve.

- Trastorno severo.

- Estudiante en silla de ruedas.

Ante estos casos, se espera que el sistema escolar funcione adecuadamente para una inclusión adecuada del estudiante en la escuela. En los casos asociados, las respuestas dadas por la institución pueden agruparse en las siguientes categorías:

- Satisfactoria, ya que tienen años trabajando en la institución.

- Institución y docente en conjunto dieron seguimiento conjunto al estudiante.

- El alumno desertó. 
- Se estableció un plan de refuerzo.

- No se hizo nada y el alumno la "va llevando".

- $\quad$ Se le ayuda con tutor.

- Actividades integradoras escuela-casa.

\section{Conclusiones}

Aunque poseen un centro de recursos para la inclusión, existen docentes que manifiestan estar en desacuerdo con la presencia de los niños, niñas y adolescentes con problemas de aprendizaje en el Centro Escolar República de Haití; se piensa que no se les puede atender adecuadamente y que su convivencia con los otros niños, niñas y adolescentes puede traer consecuencias negativas para ellos, tales como reducir el ritmo de su aprendizaje y bullying.

Por otra parte, la percepción de que existe falta de capacitación en áreas priorizadas, como evaluación de los aprendizajes, metodologías activas de atención a la diversidad y metodologías de atención específica a los diferentes trastornos de aprendizaje, es palpable entre los profesores, algunos en el área particular de formación requerida para tratar con niños, niñas y adolescentes con estos problemas, esto aunado a que no existe mucha voluntad para formarse en atención a estos estudiantes en el área de educación primaria.

Se detecta que el sistema de inclusión necesita un mayor énfasis en la formación de competencias de didáctica inclusiva y de actitud por parte de los docentes. Los niños son parte del grupo de clases, pero en algunos casos son concebidos como ajenos a estas, lo cual no cumple con lo estipulado por la Política Nacional de Inclusión.

\section{Referencias}

American Psychological Association. (s. f.). Clinical neuropsychology. Recuperado de http://www.apa.org/ ed/graduate/specialize/neuro.aspx

Arismendi, M., Primero, G., Tabullo, A., Vanotti, S. y Yorio, A. (2007). Aspectos neurofisiológicos y neuropsicológicos del aprendizaje de categorías. Revista Argentina de Neuropsicología, 9, 01-18. Recuperado de https://ri.conicet.
gov.ar/bitstream/handle/11336/27170/CONICET_Digital_ Nro.2c78aad9-8393-4b60-9ee4-8a7ec694f979_A. pdf?sequence $=2 \&$ isAllowed $=\mathrm{y}$

Crosso, C. (2014). El derecho a la educación de personas con discapacidad. Impulsando el concepto de educación inclusiva. Revista Latinoamericana de Educación Inclusiva, 79-95. Recuperado de http://repositoriocdpd.net:8080/ bitstream/handle/123456789/413/Art_CrossoC_ DerechoEducacionPersonas_2010.pdf?sequence $=1$

Ecklund-Johnson, E., \& Pearson, C. M. (2019). Foundations of contemporary neuropsychology. In C. M. Pearson, E. Ecklund-Johnson \& S. D. Gale (Eds.), Neurosurgical neuropsychology: The practical application of neuropsychology in the neurosurgical practice (pp. 2737). Recovered from https://www.sciencedirect.com/ science/article/pii/B9780128099612000035

Inclusión international, s.f. Inclusión educativa. Disponible en http://www.inclusioneducativa.org/ise.php?id=1

Ministerio de Educación de El Salvador. (MINED) (2010). Política de educación inclusiva. San Salvador, El Salvador:

Ministerio de Educación. (2019). Programa de educación inclusiva. Recuperado de https://www.mined.gob. sv/programas/programas-educativos/item/5488programa-de-educacion-inclusiva.html

Montero, I., \& León, O. G. (2007). A guide for naming research studies in psychology. International Journal of Clinical and Health Psychology, 7(3), 847-862.

Organización de las Naciones Unidas. (ONU.) (2007). El derecho de las personas con discapacidad. New York, Estados Unidos de América:

Padilla Muñoz, A. (diciembre, 2011). Inclusión educativa de personas con discapacidad. Revista Colombiana de Psiquiatría, 40(4), 670-699.

United Nations Educational, Scientific and Cultural Organization. (2005). Guidelines for inclusion: Ensuring access to education for all [versión de Adobe Acrobat Reader]. Recuperado de http://www.ibe.unesco.org/sites/ default/files/Guidelines_for_Inclusion_UNESCO_2006.pdf 